\title{
O grau de inovação das cooperativas do agronegócio da região nordeste do Estado do Rio Grande do Sul - Brasil e a construção de um framework estratégico para sustentação da inovação
}

Innovation level of cooperative agribusiness the northeast region of the State of Rio Grande do Sul_- Brazil and construction of a framework for strategic support innovation

\author{
Oberdan Teles Silva', Vitor Francisco Dalla Corte ", Carlos Alberto Oliveira \\ Oliveira 'I', Felipe Weissheimer Ferrari ${ }^{\text {IV }}$ \\ 'Imed-Instituto Meridional. oberdanteles@hotmail.com \\ "Imed-Instituto Meridional.vitor.corte@imed.edu.br \\ III UFRGS - Universidade Federal do Rio Grande do Sul. carlos.oliveira.agri@gmail.com \\ IV Imed-Instituto Meridional. fwferrari@hotmail.com
}

\section{RESUMO}

A pesquisa teve como objetivo avaliar o grau inovativo de cooperativas do agronegócio da região nordeste do Rio Grande do Sul, Brasil, por meio do Radar de Inovação, de Tidd, Bessant e Pavitt (2008). O índice de inovação efetivo das cooperativas, que fora obtido por meio de questionários preenchidos pelos gestores das cooperativas selecionadas, e inclui a mensuração da estratégia, processo, organização, aprendizagem e relacionamento, é de $72,40 \%$. Entre os construtos que obtiveram os maiores escores, estão o relacionamento $(76,23 \%)$, organização $(75,49 \%)$ e aprendizagem $(73,86 \%)$. Os construtos estratégia e processo obtiveram os menores índices, $68,77 \%$ e $67,71 \%$, respectivamente. Para potencializar os limitantes do grau inovativo, propõem-se um teamwork diversificado e reduzido, a partir dos eixos sistemas de controle formal e rotina de alto nível, ambos sustentados pela competência organizacional. O framework é limitado às cooperativas do estudo, não devendo seus resultados ser extrapolados no escopo temporal e geográfico. Pesquisas adicionais poderiam mensurar a eficiência do framework após sua implementação gerencial.

Palavras-chave: cooperativas, radar da inovação, teamwork. 


\section{ABSTRACT}

The survey had the objective of evaluating the innovative degree of agribusiness cooperatives in the northeastern region of Rio Grande do Sul, Brazil, through the innovation radar of Tidd, Bessant and Pavitt (2008). The effective innovation index of the cooperatives, which had been obtained through questionnaires completed by the managers of the selected cooperatives and includes the measurement of the strategy, process, organization, learning and relationship, is $72.40 \%$. Among the constructs that obtained the highest scores are the relationship (76.23\%), organization (75.49\%) and learning (73.86\%). The strategy and process constructs had the lowest indexes, $68.77 \%$ and $67.71 \%$, respectively. To enhance the limitations of the innovative degree, a diversified and reduced teamwork is proposed, based on the axes of formal control systems and high level routine, both supported by organizational competence. The framework is limited to study cooperatives, and its results should not be extrapolated in the temporal and geographical scope. Additional research could measure the efficiency of the framework after its managerial implementation.

Keywords: cooperatives, innovation radar, teamwork.

\section{INTRODUÇÃO}

A competitividade nos agronegócios é dependente das estruturas colaborativas, dado as pequenas organizações não conseguirem inovar em termos de modelos de gestão em decorrência das capacidades limitadas de expansão e investimentos de ordem financeira e estratégica. Somam-se a isso, os aumentos dos custos de produção, a pressão por necessidades de economias de escala e elevação da competição, devido à queda das barreiras protecionistas (VERHEES et al., 2012). O agronegócio, apesar dessas limitações, vem apresentando interesse em desenvolver capacidade de inovação e empreendedorismo por meio de relações estratégicas com diversos agentes endógenos e exógenos (WAAL; MEINGAST, 2017).

Entre as práticas das organizações dos agronegócios, referentes aos elementos internos e externos, estão as ações colaborativas, novos formatos, como as cooperativas, com vistas a relacionar-se com atores estratégicos como universidades, 
grupos de apoio, associações de negócio e instituições de nível macro, meso e micro, em busca da inovação material (DAGNINO et al., 2015). Sendo a inovação um processo colaborativo endógeno e exógeno, as cooperativas, a partir da premissa de múltiplas interações, apresentam condições de se estruturarem selecionando e adaptando estruturas em favor desta competitividade, bem como fomentando o desenvolvimento econômico (BALLAND, 2012).

As cooperativas são agentes econômicos que geram valor ao produto, além de ampliarem a renda e a empregabilidade no país. Representam a livre associação entre agentes com vistas a satisfazerem necessidades sociais, econômicas e culturais, por meio de uma empresa democrática (INTERNATIONAL CO-OPERATIVE ALLIANCE, 2015). As organizações cooperativas podem ser consideradas como alternativas para preencher lacunas das empresas com fins lucrativos, das empresas estatais e das organizações sem fins lucrativos (NOVKOVIC, 2008). No seu estudo, Vieta (2010) propõe o modelo cooperativo como uma ferramenta útil para o desenvolvimento econômico. O papel social das cooperativas, para alívio a pobreza foi o que Birchall e Simmons (2007) destacaram. A multifuncionalidade da organização cooperativa é notável desde o ponto de partida da implementação com a Sociedade dos Probos de Rochdale, em 1844. A proposta dos tecelões autônomos na Inglaterra tinha como objetivo efetuar atividades econômicas e sociais para melhorar o bem-estar dos seus membros (WILSON; SHAW; LONERGAN, 2012).

Os valores das cooperativas abarcam solidariedade, igualdade, democracia, responsabilidade social, assim como ética e honestidade. Ademais, as cooperativas são o elo entre os agronegócios e o mercado, exercendo importante papel enquanto agentes integradores locais e maximizadores da competitividade setorial (RIBEIRO et al., 2013). As cooperativas geram interações de cooperação e solidariedade, fazendo do Brasil uma das potências mundial na produção de commodities. A transposição para uma competitividade mais estratégica é dependente, contudo, do aprimoramento da inovação. A inovação no setor do agronegócio é o produto das 
atividades de pesquisa e desenvolvimento [P\&D] e dos avanços tecnológicos. Suas métricas de mensuração englobam deslocamentos positivos na função produção em termos de ganhos de escala e reduções expressivas de custo (AGUIAR; PINHO,1998). A inovação aliada aos avanços tecnológicos também se refere à introdução do desenvolvimento de novos produtos nas unidades de produção. Ao desenvolverem atividades de P\&D com os diversos atores, as cooperativas solucionam restrições presentes de mercado ampliando suas ações estratégicas em novos mercados e demandas.

No estudo, aplicou-se o instrumento denominado Radar da Inovação, de Tidd, Bessant e Pavitt (2008), junto a decisores de cooperativas do nordeste do Rio Grande do Sul. Nesse sentido, o decisor_é aquele que observa e define escolhas por meio de informações (LÖBLER; HOPPEN, 2005). Condiz com a resolução de problemas em um conjunto de alternativas. Os decisores são, portanto, aqueles que convergem comportamento em ações, sejam estas relacionadas a fatores políticos ou racionais (BATAGLIA; SIN OIH YU, 2008). No contexto deste trabalho, e em sintonia com Mises (2015), entende-se ainda como decisor, aquele que reconhece uma insatisfação momentânea e que constrói cenários futuros que sustentam suas ações direcionadas. Portanto, decisores, no âmbito das cooperativas, são aqueles que ocupam assento nas decisões hierárquicas das cooperativas em seu quadro diretivo.

O referido instrumento de verificação quantitativa da inovação mensurou os construtos estratégia, processo, organização, aprendizagem e relacionamento das cooperativas em estudo. Compreender a inovação no contexto do sistema cooperativo é importante dado o incremento nas cooperativas da utilização de estratégias de fidelização dos cooperados, das novas formas de capitalização e estratégias de governança, que visam ampliar o portfólio, o controle e a influência das cooperativas na construção de valor junto ao mercado (PIVOTO, 2015). A aplicação do Radar da Inovação teve como objetivo evidenciar, em um espaço temporal, o grau de 
inovação das cooperativas da amostra em termos de efeitos resultantes dos processos dos decisores quanto à gestão dos respectivos constructos.

Sabe-se, contudo, que além da mensuração, isto é, da explicitação do efeito dos respectivos construtos, torna-se necessário desenvolver às cooperativas um novo modelo de gestão de negócio que sustente a inovação material e intangível das mesmas. O estudo apresenta relevância diante dessa proposição: mensuração do grau de inovação em relação ao efeito tangível das ações dos gestores a partir de Tidd, Bessant e Pavitt (2008), bem como a proposição de um modelo de gestão de negócio baseado nos pilares do empreendedorismo corporativo e das redes institucionais e organizacionais, com vistas a potencializar a inovação por meio da causa, ou seja, de um modelo que sustente e amplie estes construtos.

Tendo a finalidade, portanto, de potencializar e sustentar o grau de inovação a partir destes construtos que compõem a auditoria a partir de Tidd, Bessant e Pavitt (2008), e tendo conhecimento da necessidade de além de mensurar, propor os fundamentos estratégicos desta, estruturou-se, após a análise empírica, um framework que servirá de base para aferir a teamwork competence das cooperativas, situadas em um município, mas como conexões na região de abrangência da pesquisa. O framework é estruturado a partir dos princípios do empreendedorismo corporativo e suas variáveis endógenas em termos de formatação do núcleo decisório e de sua capacidade de absorção e interações junto às redes organizacionais representadas pelas redes institucionais e organizacionais, em termos de nível macro e meso. 


\section{REFERENCIAL TEÓRICO}

\subsection{Gestão de cooperativas e o Teamwork estratégico}

Diante das modalidades de organizações mais conhecidas, os principais pontos de diferenciação das cooperativas são objetivo, propriedade, governança e financiamento. Comparando o propósito organizacional, a empresa de capital aberto é criada principalmente com o objetivo de gerar retornos positivos para seus investidores, os quais são obtidos maximizando os lucros (CHADDAD e COOK, 2004). No caso da organização cooperativa, o objetivo geralmente está focado em fornecer benefícios econômicos e sociais aos seus cooperados. No aspecto decisório, a natureza democrática de governança da cooperativa fica destacada, onde o modelo "uma ação, um voto" das empresas de capital aberto é substituído pelo modelo "um membro, um voto". Dessa forma o poder decisório em uma empresa de capital aberto geralmente recai sobre o tamanho da participação dos acionistas. Isso por si só, incentiva que as decisões estratégicas dentro das empresas de capital aberto geralmente são determinadas com base no que irá gerar os melhores retornos para o capital investido. Já nas cooperativas, a função dupla (social e econômica) coloca a organização no desafio de operar de maneira eficiente para gerar um superávit financeiro e operar de maneira sustentável, sendo a retenção de resultados suficientes para continuar a prestar serviços aos cooperados (MOONEY, 2004).

Apesar das características favoráveis, as organizações cooperativas também contam com desafios ou fraquezas relacionadas ao seu modelo de negócios que já foram identificados (COOK, 1995; ORTMANN e KING, 2007). Os conflitos organizacionais das cooperativas podem contribuir positivamente, desde que estes elementos convirjam para a competitividade por meio da negociação e divisão isonômica dos recursos limitados. O conflito nas cooperativas deixa de ser estratégico quando os indivíduos apresentam traços de personalidade como agressividade, dominância e autoritarismo. Estes fatores são típicos da impulsividade, e resultam em ações de curto prazo às custas de um futuro comprometido pelas ações nefastas destas atitudes. Contudo, o 
conflito torna-se estratégico quando os indivíduos apresentam qualidade de igualdade e confiança (MUKHERJEE; UPADHYAY, 2019). A empatia, resposta emocional frente ao outro, e um dos fatores que conduz à igualdade, contribui para o gerenciamento de conflito e para a cooperação (DAVIS, 1983). Sendo assim, as atitudes representam predições de comportamento humano, além de contribuírem com o comportamento cooperativo (COHEN; INSKO, 2008). Torna-se necessário, portanto, compreender e entender o contexto das atitudes em favor da resolução de conflitos por meio da estruturação do teamwork estratégico.

O teamwork representa a união de decisores que se reconhecem mutuamente e que buscam a realização de um propósito comum. Seus integrantes apresentam dinamismo e capacidade de adaptação, estando voltados para indicadores de desempenho. Estes indivíduos, representados por gestores contratados para gerir atividades relacionadas ao planejamento e execuções de ações operacionais e estratégicas, se caracterizam, ainda, com potencial de ação empreendedora individual (HERNANDEZ; MESA; SCHACHTER, 2018). No sistema cooperativo, podem ainda, além dos gestores, compor e apoiar o núcleo decisório do teamwork representantes dos cooperados, conselho de administração e conselho fiscal (OLIVEIRA, 2015). Estes dois últimos, como elementos responsáveis pelas decisões de gestão estratégica em atenção também às decisões das assembleias das respectivas cooperativas. São encarregados, portanto, de observar questões de curto e longo prazo (ROSA; MEDEIROS, 2007). Corroborando com esse entendimento estratégico do teamwork, Mintzberg, Lampel, Quinn e Ghoshal (2006) definem capacidade empreendedora como a busca de novos propósitos, sendo necessário que estes sejam dotados de capacidades de avaliação, técnica e de relação. A capacidade de avaliação representa a habilidade de estruturar a quantificação destas oportunidades, a técnica, por sua vez, refere-se à execução e a de relação, está direcionada à interação entre os vários agentes da cadeia de negócio. A respeito das capacidades empreendedoras do teamwork, sendo uma prática de alto desempenho, percebe-se a influência de Nelson e Winter (1982), definindo-as e relacionando-as ao gerenciamento de rotinas de alto 
nível. Conforme os autores, trata-se dos registros de informações e de registros investimento, fatores condicionantes à ampliação da competitividade da organização.

Em termos de acesso à competitividade, o teamwork deve ser formado por um núcleo decisório, em quantidade reduzida, dotado de experiência e diversidade, em termos de formação técnica, pois existe correlação de que quanto menor o núcleo decisório organizacional, maior o grau de empreendedorismo corporativo (HEAVEY; SIMSEK, 2013). Segundo esses autores, essa formatação decisória faz com que todos os processos e rotinas sejam analisados sob o prisma da mudança onde se questiona o status quo. Isso promove o questionamento da lógica reducionista da economia ortodoxa. Conduz, a partir desta premissa, a novas possibilidades de combinações estratégicas, direcionando-os aos princípios da economia criativa e também evolucionária. A diversidade, a experiência, o nível de formação e os indicadores de performance enquanto gestores, contribuem para a convergência de ideias, formulação de decisões e otimização da estrutura organizacional (HERNANDEZ; MESA; SCHACHTER, 2018).

A constante troca de experiências promove a troca e difusão de conhecimento, promovendo a geração da inovação no mercado, neutralizando o impacto das forças externas à organização. O teamwork, enquanto ápice estratégico, faz parte da governança corporativa, sendo responsável pela gestão dos recursos da organização em prol da maximização dos interesses dos stakeholders (MORÉ; GONÇALO; FIATES; ANDRADE, 2018). São responsáveis, ainda, pela correção dos desvios estratégicos, bem como de possíveis problemas futuros da organização. Apresentam capacidade de influenciar os demais atores da organização, sendo responsáveis pelos indicadores de performance e agilidade de decisões. Existe, também, uma correlação entre governança corporativa e capacidade absorvedora. A última representa a capacidade dos indivíduos do teamwork em obterem informações estratégicas que sejam convergidas estrategicamente entre processos, produtos e serviços, gerando vantagem competitiva (ZAHRA; SAPIENZA, DAVIDSSON, 2006). 
É preciso, ainda, um teamwork com capacidade de estruturar processos de controle formal, pois existe correlação positiva com inovação organizacional. Ao desenvolver as habilidades individuais relacionados a estes elementos citados, é possível estabelecer um sistema formal de controle ampliado onde se leva em consideração as tarefas desenvolvidas pelas equipes, bem como a cultura organizacional de evolução de toda a organização (SALAS et al., 2009). Isso faz com que ocorra a aprendizagem via acertos e erros, contribuindo assim, com a redescrição representacional do framework. A redescrição é a estruturação de recursos cognitivos dos decisores por meio de suas experiências e propósitos específicos (GUIMARÃES; AZAMBUJA, 2010).

\subsection{Empreendedorismo corporativo e sistemas formais de controles}

O estudo do empreendedorismo remonta a três fases: a era econômica, entre 1870 e 1940, a era das ciências sociais, entre 1940 e 1970, e a era dos estudos organizacionais, acentuando-se a partir da década de 1970 (VERGA; SILVA, 2014). A era econômica remonta aos conceitos de Cantilon, associando o empreendedorismo aos riscos e às incertezas de Frank Knight e da mudança e inovação de Joseph Schumpeter, tendo ainda o aporte dos economistas austríacos. Na era das ciências sociais, contudo, o empreendedorismo é definido a partir de traços de personalidade como cultura desviante, tendo influência em Max Weber. E na era dos estudos organizacionais, o empreendedorismo passa a ser analisado com aporte da antropologia, sociologia, psicologia e da economia. A partir destas três eras, infere-se a existência de uma correlação entre os conceitos de empreendedorismo e estratégia, pois ambos têm como fundamentação uma abordagem social, cognitiva e de transformação (ANSOF, DECLERCK, HAYES, 1990; BESANKO, 2006). Nesse sentido, o empreendedorismo refere-se à capacidade de internalidade do empreendedor em criar novas visões agrupando recursos para esta ação (FILLION, 1999). 
A partir destes elementos cognitivos, sociais e de transformação, o empreendedorismo corporativo estratégico é o responsável pelo acesso, criação e otimização de mercados, produtos e performance de inovação (GOODALE; KURATKO; HORNSBY; COVIN, 2001). O empreendedorismo tem como antecedentes a estruturação de um modelo de gestão que gere novas formas de negócio, bem como o estabelecimento de uma eficiente arquitetura de receitas e despesas (TEECE, 2010). Nesse sentido, o empreendedorismo refere-se à captura e à entrega de valor a segmentos rentáveis economicamente à organização por meio da geração da vantagem competitiva (BESANKO, 2006). Segundo o autor, o empreendedorismo deve ser visto também a partir das microfundações das capacidades dinâmicas, representando o estiramento da competência organizacional. Essas competências devem apresentar raridade, imobilidade e heterogeneidade frente aos demais competidores, devendo ser gerenciadas sob o prisma da alavancagem, combinação e reconfiguração (TIDD; BESSANT; PAVITT, 2008).

O empreendedorismo corporativo tem conotação de transformação de recursos genéricos em específicos, dado o escopo das operações estar voltado à renovação do processo estratégico (ANSOF; DECLERCK; HAYES, 1990; GINSBERG, 1990). Com vistas a maximizar essas ações estratégicas, é necessário que o modelo de gestão contemple apoio gerencial, indicadores de ação e capacidade avaliativa. Ao utilizar sistemas formais de controle junto com o papel da comunicação, se fortalece a cultura de coordenações de ações por meio da explicitação do conhecimento individual ao conhecimento sistêmico.

Os sistemas formais de controle denotam, portanto, fatores culturais como antecedentes dos sistemas de gerenciamento dos padrões organizacionais (CRESPO; RODRIGUES; SAMAGAIO; SILVA, 2019). A cultura organizacional também influencia nos sistemas controles, variando de acordo com o setor em que a organização atua, bem como da estrutura da organização, seja ela mecânica ou orgânica (LANGFIELD-SMITH, 
2007). A estrutura orgânica da organização está voltada a incertezas do mercado. Até a década de 90 , os sistemas formais de controle estavam voltados a indicadores contábeis. Atualmente, os sistemas de controle organizacional contemplam gestão de recursos humanos, financeiro, vendas e estratégia (DAVILA; FOSTER, 2007). Os aprimoramentos dos indicadores de controle também estão direcionados aos sistemas de feedback, que auxiliam a organização na sua convergência ou reorientação estratégica. Estas ações de gerenciamento estratégico da organização podendo ser direcionadas em custo, diferenciação ou enfoque, conforme Porter (1999) (LANGFIELD-SMITH, 2007). A primeira, em custo, deve utilizar ênfase em sistemas centrais de controle, e a segunda em sistemas integrados.

Em termos de sistemas de controle e empreendedorismo, percebe-se a relação conceitual com orientação estratégica, em virtude da relação com os princípios que influenciam o comportamento da firma em favor da garantia da viabilidade do negócio e da performance (CACCIOLATTI; FEARNE, 2013). Os sistemas formais de controle, nesse sentido, objetivam garantir a performance tanto financeira quanto de inovação da organização (GRINSTEIN, 2008). Também devem garantir a obtenção de padrões de qualidade pré-estabelecidos pela governança corporativa (LANDER; REINSTEIN, 2005). Nesse sentido, a orientação estratégica está alicerçada na orientação ao cliente, que compreende a identificação das necessidades dos clientes, gerando valor superior, apresentando correlação com a performance baseada em receitas e inovação (GRINSTEIN, 2008).

Os sistemas de controle também apresentam conexão com a governança corporativa, pois são estes sistemas que asseguram a performance financeira aos acionistas através da alocação de recursos escassos, com vistas a gerar valor e produtividade (LANDER; REINSTEIN, 2005). Do mesmo modo, esse sistemas asseguram a correta alocação de processos e estruturas que maximizam o retorno financeiro superior. Isso é operacionalizado pelo alinhamento de valor econômico, 
que deve garantir que os custos de capitais sejam cobertos. Assim, os sistemas de controle são responsáveis pela mensuração de todos os fatores de produção.

Os sistemas de controle também estão relacionados à competência gerencial e competência de liderança (LANDER; REINSTEIN, 2005). A primeira condiz com o alinhamento estratégico entre gestão de pessoas e capacidades de mobilizar recursos em favor das metas da organização. A segunda refere-se à capacidade das pessoas em gerar e se adaptar a mudanças a partir da execução da missão e da visão organizacional. Destaca-se que esses sistemas de controle devam estar relacionados ao apoio das redes institucionais e organizacionais.

2.3 O papel das redes institucionais, organizacionais e a capacidade de absorção

As cooperativas dos agronegócios se caracterizam como um sistema integrado de mercados que conectam vários agentes, podendo assim, serem analisadas à luz das redes onde são pesquisados processos de inovação e integração (GAZDECKI, 2018). Entende-se por inovação, desde o aprimoramento de produtos e serviços até a geração e disseminação de conhecimentos compartilhados em favor de ampliação da competitividade (KAWAMOTO; SPERS, 2019). As interações das redes podem ser analisadas sob os pontos de processos e seus participantes. Nesse ambiente ocorrem os fluxos de interações propiciando uma atmosfera estratégica (HAKANSSON, 1982). Nestas redes, se desenvolvem as interações de curto prazo e as de longo prazo. A primeira é regida pelo fluxo monetário e trocas sociais, e a segunda difere por relações estratégicas entre empreendimentos e ajustes mútuos de processos. Ambas geram fluxos de eventos e, consequentemente, conjunto de ações, sejam estas em termos de solução de problemas, penetração de mercado e gestão de inovação. Alta qualidade de interações nas redes, representados por confiança, comprometimento, satisfação, adaptação geram externalidades positivas em termos financeiros (HALINEN et al., 2013). 
As redes que contribuem para a capacidade de absorção e gestão da inovação classificam-se, também, em organizacional e institucional. A organizacional, também denominada de rede de mercado, é constituída pelo agrupamento entre fornecedores, clientes e competidores (KIM; LUI, 2015). Nesse tipo de rede, os agentes econômicos desenvolvem conhecimento tácito ou subjetivo, estando estritamente relacionado à demanda em que atuam. O conhecimento, portanto, está inserido nas atividades de produção de cada agente econômico. Isso torna complexo o processo de codificação do conhecimento, dado o interesse competitivo das organizações. Percebe-se que alta competitividade de cada firma faz com que ocorra o desenvolvimento de objetivos utilitaristas de cada um dos membros da respectiva rede.

A rede institucional, entretanto, envolve agentes de fomento, órgãos governamentais, universidades e institutos de pesquisa. Esta rede também é tipificada como uma rede de exploração. Visa, em sua essência, o desenvolvimento de novas ideias e oportunidades voltadas para o descobrimento de avanços tecnológicos. Em termos de conhecimento, estas redes institucionais apresentam informações que se encontram dispersas no mercado. Além disso, os atores das redes institucionais produzem conhecimento técnico e tecnológico superior a outras formas de redes (KIN; LUI, 2015).

A proximidade cognitiva dos atores, decorrentes das dimensões não espaciais e espaciais entre as cooperativas e as redes, sejam elas institucionais e organizacionais, são determinantes positivos das externalidades de competitividade das cooperativas relacionadas ao agronegócio (GELDES; HEREDIA; FELZENSTEIN; MORA, 2017). Ademais, isso também faz, conforme os respectivos autores, que a cooperação organizacional apresente determinantes positivos relacionados à inovação. As proximidades, elementos que facilitam a inovação através da absorção e troca de conhecimentos, abarcam elementos de cognição, que se refere à divisão mútua de referências e 
conhecimento. Já a proximidade geográfica, compreende a localização física dos atores. E a proximidade organizacional, por sua vez, refere-se à simetria de relacionamentos mantidos. A proximidade de tecnologia aplicada e a institucional englobam regras e rotinas que facilitam ações colaborativas (BOCHMA, 2005).

A cooperação para inovação, por meio das redes e suas interações, representa a ação ativa entre diferentes agentes que buscam realizar inovações em atividades de gestão, sejam eles fornecedores, clientes, concorrentes, agentes públicos e universidades. A cooperação destas relações em rede, em favor da gestão da inovação, representa o ajuste simétrico dos diversos inputs entre os agentes, sejam estes indivíduos, equipes, parceiros e concorrentes. Todos esses contribuem para a divisão de custos e know how mútuo (DODGSON; ROTHWELL, 1994). As ações de cooperação em rede apresentam, ainda, foco na territorialidade, tendência à sustentabilidade ambiental, social e econômica, e orientação ao mercado. A constituição das cooperativas apresentam tendências à gestão estratégica e de inovação (MOURA; MADEIRA; DUARTE; CARVALHO; KAHILANA, 2019). A gestão da inovação tem como antecedentes múltiplas interações, adicionando em seus processos novos conhecimentos e recursos econômicos no mercado (WALTERS; RAINBIRD, 2007). As atividades de cooperação para inovação contemplam a troca de informações entre os agentes, via redes, materializando, consequentemente, a capacidade de absorção das cooperativas. A capacidade de absorção é entendida por Tortoriello (2015) como a habilidade de compreender, adquirir, usar e, finalmente, tomar vantagem do conhecimento disponível fora da organização. A capacidade de absorção tem dependência da trajetória, sendo que as organizações que implementam P\&D são aquelas que otimizam o uso da informação externa podendo apresentar maior proatividade frente às oportunidades. Ademais, a capacidade de absorção depende da capacidade dos indivíduos da organização e da estruturação de comunicação entre o ambiente externo e a organização (COHEN; LEVINTHAL, 1990). A conjunção de fatores até mesmo elementares faz com que ocorra uma amplitude infinita de variedades, típica da inovação (SIMON, 1981). 
Isso resulta ainda em spillover e atividades de pesquisa e desenvolvimento contribuindo, também, com a complementaridade dinâmica. Proporciona a adição de novos conhecimentos exógenos na estratégia da organização (LOVE et al., 2014). O conhecimento, que se origina nas interações intra e interorganizacional, através da capacidade de absorção, faz com que os recursos da organização se transformem em serviços estratégicos (PENROSE, 2008). A referida autora compara o conhecimento como propulsor dos ativos da organização, que quando combinados prestam serviços administrativos ou técnicos. Tem-se, contudo, como fundamentação destes serviços a gestão eficiente de aptidão de coordenação, contribuindo ainda para a aprendizagem organizacional das cooperativas.

\subsection{Cooperativas e aprendizagem organizacional}

A aprendizagem organizacional representa a interação que gera interpretação e implementação de conhecimentos nos diversos níveis organizacionais. Interações e práticas de aprendizagem cooperativas conduzem a capacidade de inovação. Existe correlação entre trajetória de pesquisa e desenvolvimento das cooperativas com interações com universidades (COSTA; PORTO; SILVA, 2003). Segundo eles, quanto maior a experiência em cooperação, maior a tendência para geração de performance em inovação. A experiência em cooperação também é relevante, pois a aprendizagem aprimora os fluxos de interações promovendo novas realocações dos recursos organizacionais (LAVIE; ROSEMKOPF, 2006).

Para Costa, Porto e Silva (2003), quanto maior o investimento em P\&D e interações de cooperação, maior a performance tecnológica das organizações. Requerem, contudo, uma equipe de trabalho competente que gerencie esta coordenação. Essa equipe atua na divisão das tarefas, de informação e soluções de problemas. Estudos comprovam que a aprendizagem cooperativa atua positivamente 
nas dimensões do teamwork competence, como eficácia coletiva, planejamento, estabelecimento de metas, soluções de restrições e gerenciamento de conflitos.

As práticas de aprendizagem cooperativa referem-se, também, ao desenvolvimento de competências transversais e coletivas dos indivíduos junto à competência do trabalho em equipe. Neste sistema, o indivíduo é responsável pelo desenvolvimento de suas habilidades, mas também auxilia na construção coletiva de habilidades sociais, interações, bem como da reflexão do grupo. É mister que a aprendizagem cooperativa primeiramente seja direcionada ao desenvolvimento das habilidades individuais relacionadas à capacidade de estabelecimento de metas, planejamento e gerenciamento de conflitos.

A aprendizagem organizacional em rede tem como antecedentes 0 estabelecimento de uma estrutura de governança corporativa que seja adequada para as interações estratégicas entre as organizações. Esta estrutura deve ser institucionalizada contemplando o gerenciamento de uma rotina que viabilize a troca de habilidades e conhecimento (COSTA; PORTO; GALINA; PISCOPO; MACCARI, 2017). Dessa forma, será possível transferir, recombinar e criar novos conhecimentos. A gestão de aprendizagem ainda é condicionada à capacidade absorvedora de cada organização. Entre os elementos que facilitam a gestão da aprendizagem estão a contribuição ativa entre os parceiros, o ajustamento de objetivos entre os decisores de cada organização e a efetiva comunicação sobre o processo de cooperação e monitoramento das interações. 


\section{PROCEDIMENTO METODOLÓGICO}

Constituíram a pesquisa o total de cinco cooperativas do setor do agronegócio situadas na região nordeste do estado do Rio Grande do Sul, no município de Sananduva, porém, com conexões de negócio em toda a região, contemplando mais de 20 municípios. O estudo, de natureza quantitativa e descritiva, foi desenvolvido por meio de questionários, que foram aplicados diretamente a cada um dos gestores das cooperativas, selecionadas por conveniência. Uma das ferramentas utilizadas para mensuração do grau inovativo das organizações foi desenvolvida por Tidd, Bessant e Pavitt (2008), denominada Radar da Inovação. Em pesquisa realizada na Scielo sob o termo, radar da inovação, constatou-se o total de dois artigos publicados nos anos de 2014 e 2017, nas áreas temáticas da ciência social interdisciplinar e comunicação. Já na base de periódicos da Coordenação de Aperfeiçoamento de Pessoal de Nível Superior [CAPES], identificou-se, entre os anos de 2004 e 2017, o total de 159 artigos divididos em áreas de gestão, engenharia, tecnologia e materiais.

Mensurou-se, neste instrumento de coleta, os indicadores referentes à estratégia, processos, organização, relacionamentos e aprendizagem, no modelo proposto por Tidd et al. (2008). Para cada construto, o gestor deveria assinalar o grau de concordância com oito afirmações, em um total de 40 afirmações, sem a identificação de a qual construto a sentença se referia. As sentenças descreviam padrões de comportamento (vide apêndice 1), tais como: "As pessoas têm uma ideia clara de como a inovação pode nos ajudar a competir". As respostas, estruturadas em uma escala do tipo Likert, variavam entre 1 - "definitivamente falso", que representa a não adesão absoluta à prática, e 7 - "muito verdadeiro", que representa a adesão integral àquela prática e é considerada a ideal pelos autores. Os números 2 ao 6 , representavam níveis intermediários de adesão, que iam de "falso" até "verdadeiro". Embora, semanticamente, verdadeiro e muito verdadeiro possam ter o mesmo significado, a estruturação em uma escala deixa claro que um está acima do outro, ainda que não se elimine a subjetividade. 
Um dos elementos relevantes da auditoria é a possibilidade de quantificação de cada um dos construtos, em termos de otimização de sua utilização e quantificação de insuficiência, bem como explicitação dos pontos mais estratégicos de cada um dos indicadores. A figura 01 a seguir demonstra, de forma sintética, os principais elementos mensurados em cada um dos construtos da auditoria da inovação.

Figura 01 - Construtos do instrumento de auditoria inovadora

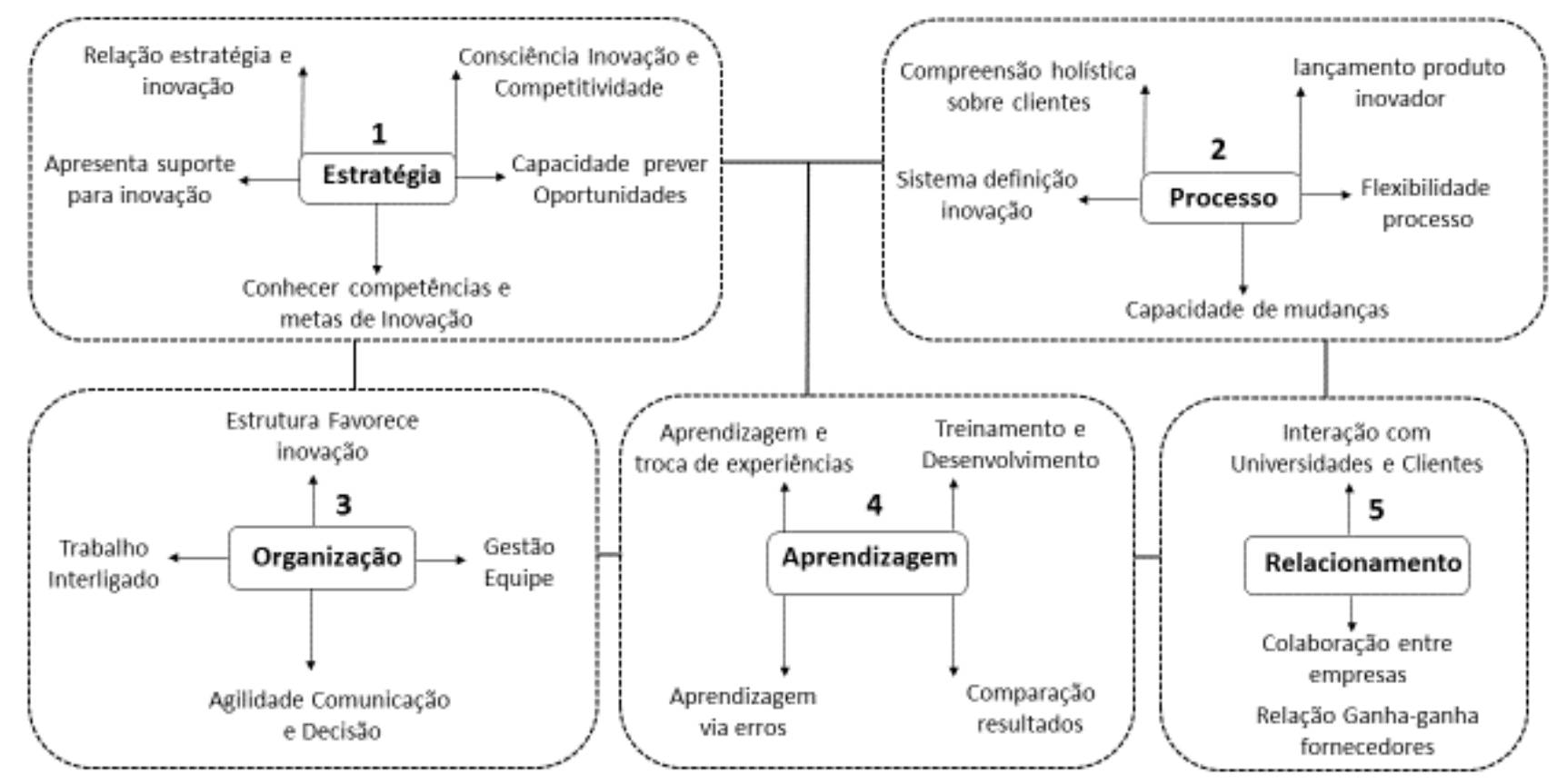

Fonte: adaptado partir de Tidd, Bessant e Pavitt (2008)

No construto estratégia (1), é verificado se a estratégia da organização sustenta a inovação, se existe a consciência de que a inovação gera a competitividade, bem como se esta auxilia na previsão de novas oportunidades mercadológicas. Tem-se a finalidade, ainda, de identificar a relação entre competências e metas de inovação, e se este construto promove a inovação. 
Em termos de gestão de processos (2), a auditoria explicita se a organização possui compreensão holística junto aos clientes, onde o processo é caracterizado como um sistema de inovação promovendo o lançamento de um novo produto. Verifica-se ainda neste quesito, se os processos geram mudanças por meio da flexibilidade. No fator organização (3), o instrumento aponta se a estrutura favorece a inovação e a gestão de equipes por meio de processos interligados. Mensura, também, o processo de agilidade e flexibilidade da comunicação e da decisão organizacional.

No construto aprendizagem (4), o instrumento demonstra se ocorre na organização a aprendizagem via erros por meio da comparação de resultados perante os demais competidores. Também aponta se ocorre a aprendizagem por troca de experiências, e se o fluxo de aprendizagem desenvolve práticas de treinamento e desenvolvimento de pessoas. E em termos de construto relacionamento (5), tem-se a verificação se a organização interage com universidades e clientes em favor da ampliação da competitividade, e se existe, ainda, relações ganha-ganha entre organizações e fornecedores.

Os dados foram analisados por meio de estatística descritiva, sendo mensurado o índice inovativo de cada cooperativa, e de cada um dos construtos, individualmente. A análise dos dados permitiu a identificação dos elementos limitantes de cada construto. Considerou-se para tanto, o índice de até de 3,5 como insuficiente, que corresponde a $50 \%$ de efetividade inovadora. Contudo, naqueles construtos onde a mensuração fora superior a 3,5, considerou-se a menor escala do construto a partir deste escore. 


\section{ANÁLISE DE RESULTADOS}

A tabela 01 sintetiza os resultados da mensuração das cooperativas objeto de estudo.

Tabela 1 - resultados dados obtidos.

\begin{tabular}{|c|c|c|c|c|c|c|c|}
\hline Estratégia & Questões & $\begin{array}{c}\text { Empresa } \\
\text { A }\end{array}$ & \begin{tabular}{|c|} 
Empresa \\
B
\end{tabular} & $\begin{array}{c}\text { Empresa } \\
\text { C }\end{array}$ & $\begin{array}{c}\text { Empresa } \\
\text { D }\end{array}$ & $\begin{array}{c}\text { Empresa } \\
\text { E }\end{array}$ & Escore/Item \\
\hline & $\overline{c 1}$ & 4,5 & $\overline{5,33}$ & 2,5 & 2,6 & $\overline{5}$ & 3,399 \\
\hline & $\bar{c} 6$ & $\overline{5,17}$ & $\bar{~} 4$ & 6 & $\overline{6,2}$ & 5,6 & $\overline{5,39}$ \\
\hline & 11 & 5,17 & 4,67 & 3,5 & 4,6 & 4,6 & 4,51 \\
\hline & 16 & 5,5 & 5,33 & 5,5 & 4,4 & 4,8 & 5,11 \\
\hline & 21 & 5,5 & 5 & 4 & 4,6 & 4,8 & 4,78 \\
\hline & 26 & 4,83 & 5 & 5,5 & 5,2 & 3,4 & 5,03 \\
\hline & 31 & 5 & 4 & 5,5 & 5,2 & 4,6 & 4,86 \\
\hline & 36 & 4,67 & 5 & 5 & 5,6 & 5,2 & 5,09 \\
\hline Média & & 5,04 & 4,79 & 4,69 & 4,8 & 4,75 & \\
\hline Média Efetiva & 4,81 & $68,77 \%$ & & A desejar & $31,23 \%$ & & \\
\hline Processos & 2 & 5,5 & 4 & 5 & 4,8 & 5,6 & 4,98 \\
\hline & 7 & 4,83 & 4,33 & 5,5 & 5 & 4,6 & 4,85 \\
\hline & 12 & 4,5 & 2,67 & 5 & 4,6 & 3,6 & 4,07 \\
\hline & 17 & 4,83 & 3,67 & 5 & 5 & 4,2 & 4,54 \\
\hline & 22 & 5,33 & 4,33 & 7 & 4 & 4,2 & 4,97 \\
\hline
\end{tabular}


21 | SILVA, O. T.; DALLA CORTE, V. F.; OLIVEIRA, C. A.; FERRARI, F. W.

\begin{tabular}{|c|c|c|c|c|c|c|c|}
\hline & 27 & 4,67 & 3,67 & 5 & 5 & 4,2 & 4,51 \\
\hline & 32 & 4,67 & 4,33 & 5 & 5,6 & 4,4 & 4,8 \\
\hline & 37 & 5 & 5,33 & 5 & 5,2 & 5,6 & 5,23 \\
\hline Média & & 4,92 & 4,04 & 5,31 & 4,9 & 4,55 & \\
\hline Média Efetiva & 4,74 & $67,71 \%$ & & A desejar & $32,29 \%$ & & \\
\hline \multirow[t]{8}{*}{ Organização } & 3 & 5,33 & 4,33 & 5,5 & 5,4 & 4,6 & 5,03 \\
\hline & 8 & 5 & 6,33 & 7 & 5 & 4,8 & 5,63 \\
\hline & 13 & 5,67 & 6,33 & 5,5 & 4,8 & 4,8 & 5,42 \\
\hline & 18 & 5 & 4 & 5 & 4,4 & 4,4 & 4,56 \\
\hline & 23 & 5,17 & 6 & 5 & 5 & 4,6 & 5,15 \\
\hline & 28 & 4,5 & 3,67 & 6 & 4,6 & 5,4 & 4,83 \\
\hline & 33 & 5,33 & 5,67 & 5 & 5,4 & 5,4 & 5,36 \\
\hline & 38 & 5,67 & 6,33 & 7 & 6,2 & 6,2 & 6,28 \\
\hline Média & & 5,21 & 5,33 & 5,75 & 5,1 & 5,03 & \\
\hline Média Efetiva & 5,28 & $75,49 \%$ & & A desejar & $24,51 \%$ & & \\
\hline \multirow[t]{6}{*}{ Relacionamento } & 5 & 6 & 5,67 & 6,5 & 5,8 & 6,6 & 6,11 \\
\hline & 10 & 4,33 & 4 & 7 & 5 & 4,6 & 4,99 \\
\hline & 14 & 5,5 & 5,67 & 7 & 4,8 & 4,8 & 5,55 \\
\hline & 19 & 4,83 & 4,67 & 5,5 & 5 & 5 & 5 \\
\hline & 24 & 5,33 & 5,33 & 6 & 5,8 & 4,4 & 5,37 \\
\hline & 29 & 6 & 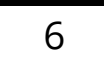 & 7 & 4,8 & 5,4 & 5,84 \\
\hline
\end{tabular}




\begin{tabular}{|c|c|c|c|c|c|c|c|}
\hline & 34 & 4,33 & 5,33 & 4 & 5,2 & 4,8 & 4,73 \\
\hline & 39 & 5,17 & 4,33 & 6,5 & 4,2 & 5,4 & 5,12 \\
\hline Média & & 5,19 & 5,13 & 6,19 & 5,08 & 5,09 & \\
\hline Média Efetiva & 5,34 & $76,23 \%$ & & A desejar & $23,77 \%$ & & \\
\hline Aprendizagem & 4 & 5,5 & 4,67 & 7 & 6,6 & 3,4 & 5,43 \\
\hline & 9 & 4,33 & 4,33 & 6,5 & 5 & 3,8 & 4,79 \\
\hline & 15 & 6 & 6 & 5 & 5,4 & 5,4 & 5,56 \\
\hline & 20 & 5,67 & 5,33 & 5,5 & 5,8 & 3,2 & 5,1 \\
\hline & 25 & 5,83 & 4,67 & 6,5 & 5,4 & 4,4 & 5,36 \\
\hline & 30 & 5,67 & 4,67 & 6,5 & 4,2 & 5,6 & 5,33 \\
\hline & 35 & 5,17 & 4,67 & 5 & 4,6 & 5 & 4,89 \\
\hline & 40 & 5,17 & 4,67 & 5,5 & 4 & 5 & 4,87 \\
\hline Média & & 5,42 & 4,88 & 5,94 & 5,13 & 4,48 & \\
\hline Média Efetiva & 5,17 & $73,86 \%$ & & A desejar & $26,14 \%$ & & \\
\hline $\begin{array}{l}\text { Total Inovação } \\
\text { Setor }\end{array}$ & 5,07 & $72,40 \%$ & & A desejar & $27,60 \%$ & & \\
\hline
\end{tabular}

Fonte: análise dos dados (2019).

No construto estratégia, identificou-se que existe uma efetividade estratégica de $68,77 \%$. Entre os fatores a serem aprimorados, que compreendem uma insuficiência de 31,23\% com vistas a atingir a elevação da métrica, estão o desenvolvimento de uma cultura organizacional que demonstre a todos colaboradores a relevância da geração de inovação para com a competitividade da 
organização. Demonstrou-se, ainda, a necessidade da apresentação e estruturação do suporte a estes processos inovadores. Além disso, apontou-se, na verificação, a necessidade das organizações em estudo conhecerem efetivamente as competências que devem ser assentadas as metas da inovação. Ao identificar a competência, é possível convergir processos de inovação com vistas a potencializar a performance da organização. Entre os destaques estratégicos das cooperativas que elevaram a métrica mensurada, estão a utilização de técnicas de previsão quantitativa referentes a oportunidades e ameaças do mercado que cercam as cooperativas.

Referente ao construto processos, obteve-se o grau de otimização das cooperativas de $67,71 \%$, sendo $32,29 \%$ de ineficiência correspondente à ausência de compreensão holística e sistêmica das necessidades demandadas pelo mercado. Desta forma, no ambiente de inovação é necessário cada vez mais buscar entender as necessidades oriundas da demanda, ou seja, a demand pull (DI STEFANO; GAMBARDELLA; VERONA, 2012). Uma das deficiências identificadas se refere também à centralização das informações mercadológicas, relativas aos clientes, no setor de marketing, não sendo permeadas aos demais setores das cooperativas. A elevação da efetividade de processos está condicionada ao conhecimento das necessidades e relevância do cliente em todos os demais setores da organização. Contudo, as cooperativas apresentam flexibilidade de desenvolvimento de produtos decorrentes de novos projetos que demandem pequenas alterações.

Em termos de organização, as cooperativas apresentaram efetividade de $75,49 \%$, sendo o trabalho em equipe um dos destaques que contribuem para este indicador. A carência de efetividade de $24,51 \%$ tem como um dos geradores a estrutura endógena hierárquica, que dificulta a tomada de decisões e fluxos de comunicação em todos os sentidos, desta forma tomar decisões no ambiente do cooperativismo, pode ter desafios de alinhar as estratégias e negócios às expectativas dos cooperados (PIVOTO et al., 2014). No relacionamento, fator que compreende interações estratégicas com fornecedores, detectou-se escore de 76,23\% de 
efetividade. A relação ganha-ganha entre cooperativas e fornecedores é um dos elementos que contribuem para esta quantificação. A limitação de $23,77 \%$ encontrase não interação entre agentes de conhecimento local e nacional, com vistas a ampliar estrategicamente o conhecimento em favor de relacionamentos mais estratégicos. No construto aprendizagem, identificou-se efetividade de $73,86 \%$, sendo a aprendizagem a partir dos erros como um dos elementos responsáveis por este índice. A limitação de $26,14 \%$, contudo, encontra-se pela demora na comparação dos resultados dos projetos executados e ajustes necessários para melhoria do desempenho.

Os resultados apontam que as cooperativas do agronegócio do nordeste do estado do Rio Grande do Sul necessitam aprimorar em termos de processos endógenos a estruturação de processos que suportem a inovação e que fomentem, consequentemente, a cultura para inovação. Um dos caminhos para criar este tipo de cultura de inovação, poderia ser a criação e gestão de estruturas de pesquisa e desenvolvimento, como uma forma de suportar este processo (CORTE; WAQUIL; STIEGERT, 2015). Identificou-se, também, a necessidade de aprimoramento do fluxo de comunicação interna por meio do reajuste da verticalização interna das cooperativas. Constatou-se, ainda, a necessidade de realização de avaliações de projetos desenvolvidos comparando e corrigindo ajustes com vistas a ampliar o desempenho. Em termos exógenos, é comum entre as cooperativas a necessidade de ampliar a compreensão sistêmica das necessidades de mercados, bem como de ampliação de relações com redes institucionais.

\subsection{Discussão geral e proposição teamwork}

Com base nos resultados empíricos, que denotam insuficiências em questões relacionadas à cultura da inovação, ausência de compreensão holística de mercado, centralização de informações e hierarquia vertical, propõem-se a estruturação de um framework estratégico direcionado à gestão das cooperativas do agronegócio com 
vistas a potencializar a competitividade por meio da redução do impacto negativo destas ações de ineficiência, conforme a figura 02.

Figura 02 - Proposição do framework estratégico às cooperativas do agronegócio.

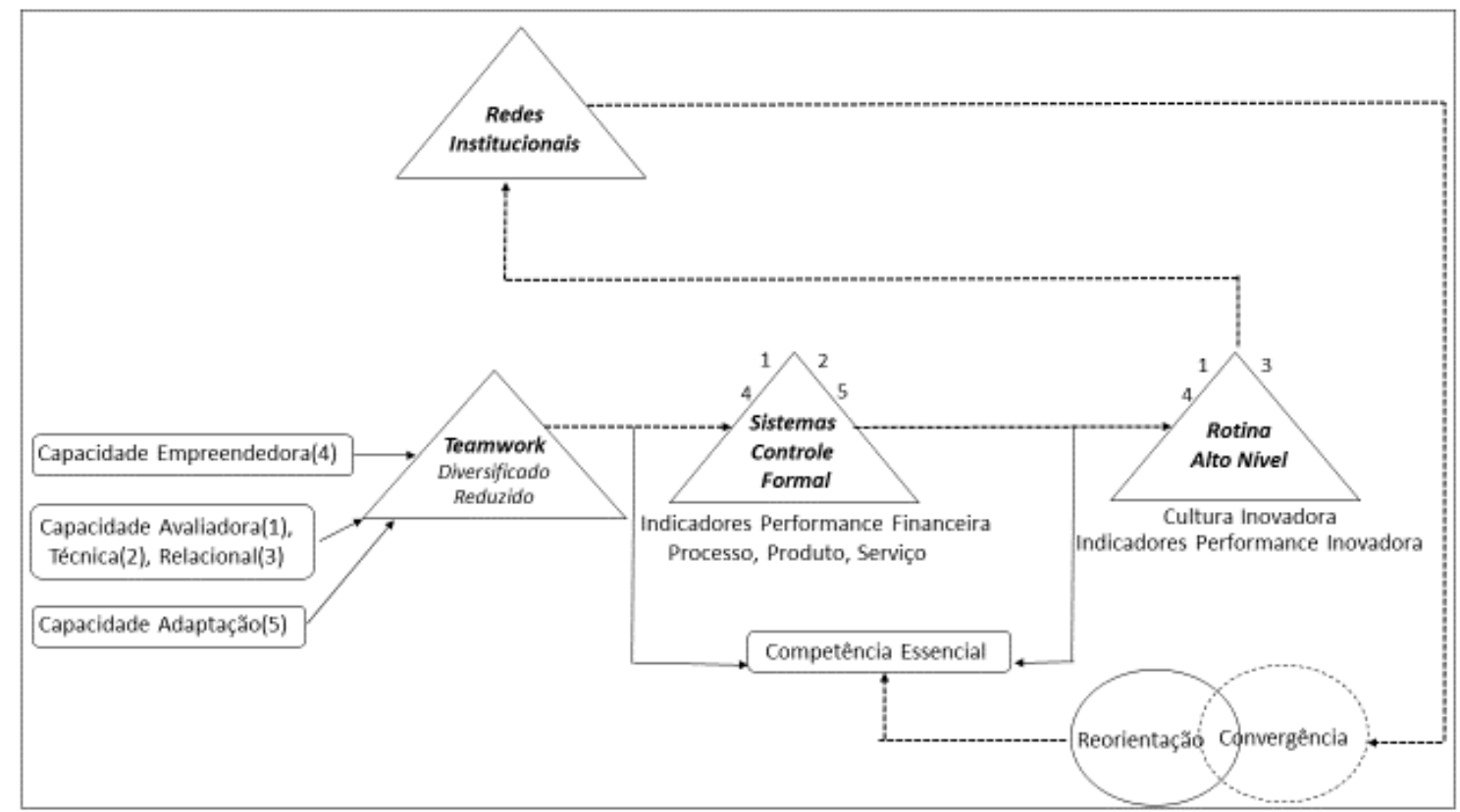

Fonte: adaptado a partir de (SCHUMPETER, 1954; SIMON, 1981; PORTER, 1999; BESANKO, 2006; LANGFIELD-SMITH, 2007; WALTERS; RAINBIRD, 2007; MINTZBERG; LAMPEL; QUINN; GHOSHAL, 2006; TIDD; BESSANT; PAVITT, 2008; HEAVEY; SIMSEK, 2013; LAMORE; BERNSTEIN; SCHWEITZER; LAWTON; RORABACK,;PERUSKI; DYKE; FERNANDES, 2013, KIN; LUI, 2015, HERNANDEZ; MESA, SCHACHTER, 2018).

A estruturação do framework tem a finalidade de servir de base conceitual à gestão estratégica das cooperativas do agronegócio. No construto estratégia, a limitação encontra-se na cultura ineficiente para inovação para que esta apresente suporte a tais processos. Demonstrou-se a necessidade da exata identificação da competência estratégica das cooperativas, com vistas a alinhar com as práticas de gestão da inovação. No construto processo, a insuficiência reside na ausência de compreensão sistêmica das necessidades do mercado, bem como na retenção de fluxos de informação de mercado na gestão do marketing, apenas. No construto organização, tem-se a necessidade de descentralização vertical, e consequente maior 
fluidez de comunicação. No item relacionamento, explicitou-se a necessidade de interação com as redes institucionais. E na aprendizagem, detectou-se a necessidade de comparação de resultados de projetos anteriores para implementação de possíveis correções.

Assim, caberá aos gestores do teamwork proporem um sistema de criação de valor responsável por além de neutralizar as deficiências detectadas, identificar fontes de oportunidades junto às redes institucionais através de elementos de valor que potencializem processos estratégicos ao modelo de negócio garantindo sustentabilidade da vantagem competitiva (WALTERS; RAINBIRD, 2007). Esse sistema de criação de valor por meio do teamwork deve estar direcionado à identificação das metas das cooperativas, definindo as oportunidades e suas respectivas valorações, para então propor gerenciamento das respectivas restrições identificadas (SIMON, 1981). Essas valorações, a priori, devem estar direcionadas aos stakeholders de investimento, clientes e parceiros.

A partir desta exposição inicial, entende-se que o teamwork, isto é, núcleo decisório, deve ser formado por um grupo de decisores diversificados e reduzido em termos quantitativos, pois existe correlação desta configuração com a gestão de inovação estratégica (LAMORE; BERNSTEIN; SCHWEITZER; LAWTON; RORABACK; PERUSKI; DYKE; FERNANDES, 2013). A diversificação refere-se a gestores com formações diferentes, não necessariamente em áreas de conhecimento correlatas. Esses gestores devem ser possuidores de capacidades avaliadora (1), técnica (2), relacional (3), empreendedora (4) e de adaptação (5) (MINTZBERG; LAMPEL; QUINN; GHOSHAL, 2006).

Entende-se que o teamwork deva gerenciar a organização a partir de duas matrizes: sistema de controle formal e rotina de alto nível. Necessário, contudo, que estes blocos operacionais e estratégicos, respectivamente, estejam alinhados de forma consciente com a competência essencial da cooperativa (TIDD; BESSANT; 
PAVITT; 2008). A matriz de sistema de controle é operacional, e está baseada nos indicadores de performance financeira e de desempenho de produtos, processos e serviços a partir de padrões de qualidade pré-estabelecidos e de estratégias, sejam de custo ou diferenciação, ou de combinação (PORTER, 1999; LANGFIELD-SMITH, 2007). Para maximizar essa matriz, é preciso que os gestores do teamwork apresentem capacidades avaliadora (1), técnica (2), empreendedora (4) e adaptação (5). A primeira, avaliadora (1), refere-se à análise dos indicadores de feedback dos processos e produtos. A técnica refere-se à implementação e correção dos desvios dos indicadores aferidos. A de adaptação (5), compreende a convergência dos processos para ampliação do potencial da competência, representada pelo seu efeito elástico empreendedor (SCHUMPETER, 1954). Já a capacidade empreendedora (4), abarca a configuração de uma nova competência decorrente das informações obtidas por meio da relação de interação entre a rotina de alto nível com as redes institucionais (KIN; LUI, 2015).

A outra, rotina de alto nível, compreende atividades de P\&D, sendo o agente fomentador da cultura organizacional inovadora e de indicadores de performance de inovação. A rotina de alto nível, representada por P\&D, influencia a cultura da organização, sendo a responsável por propor mudanças e alterações dos sistemas de controle formais e também em termos de registros de informações, de investimento, de novas técnicas e matrizes estratégicas. Por meio da capacidade avaliadora (1), relacional (3) e empreendedora (4), os gestores do teamwork mantêm interações bidirecionais e fluxos de informações ascendentes e descendentes com as redes institucionais. Por meio da capacidade avaliadora (1), isto é, a partir dos resultados dos sistemas de controles formais, identificam pontos de alavancagem, gerando capacidade de absorção de informações do mercado por meio das redes. Isso se viabiliza por meio da capacidade seletiva relacional (3). A partir disso, a capacidade empreendedora (4) fundamenta endogenamente as necessidades de convergência ou reorientação estratégica da organização. 
As contribuições do estudo para o campo em análise se referem à explicitação dos elementos que balizam as ações das cooperativas da região nordeste do Rio Grande do Sul. Entre estes, foram identificados que o construto organização, representada pela gestão de equipe e agilidade nas decisões, o construto relacionamento, signo de integração e colaboração com parceiros, e o construto aprendizagem, sinônimo de correção de erros, valorização de experiência e comparação de resultados, são fatores positivos desenvolvidos pelas cooperativas. Demonstrou-se ainda, como contribuição às cooperativas, que todos esses fatores, como organização, aprendizagem e relacionamento, são elementos propulsores da performance das cooperativas desta localidade geográfica, e devem estar assentados em processos e estratégias para sustentabilidade da performance. Para tanto, sugerese a criação de um sistema de controle formal juntamente com rotinas de alto nível, sustentadas pelas respectivas competências das cooperativas.

\section{CONSIDERAÇÕES FINAIS}

A proposição do teamwork ressalta, portanto, uma configuração direcionada a reduzir os impactos de elementos que limitam o grau inovativo das cooperativas do agronegócio em estudo. O modelo não contempla, entretanto, o aporte das redes organizacionais, pois o estudo apontou uma efetividade das cooperativas pesquisadas em termos de relacionamentos estratégicos entre os parceiros. Nesse sentido, o framework é direcionado dentro de um espaço temporal e geográfico limitado, não podendo ser estendido a outros setores ou organizações, pois compreende uma configuração específica às organizações do referido estudo. Pesquisas adicionais poderiam ser desenvolvidas após as cooperativas implementarem gestão do referido framework em seus processos gerenciais e estratégicos.

As implicações acadêmicas deste trabalho residem, portanto, na implementação de um instrumento de aferição do grau inovativo das organizações, a partir de Tidd, Bessant e Pavitt (2008), em um setor de relevância ao setor primário da 
produção do estado do Rio Grande do Sul, Brasil. Tal setor é responsável pela estabilização da balança comercial brasileira, representando $46 \%$ das exportações do país. Ademais, o agronegócio brasileiro em 2016 fora responsável por 26\% do produto interno bruto (EMBRAPA, 2018). As cooperativas, juntamente com o aporte público, vêm impulsionando a competitividade do agronegócio por meio da implementação da ciência e tecnologia, isto é, da capacitação técnica e das relações estratégicas que se desencadeiam nas trocas de informações, insumos e ciclos de produção.

A relevância da pesquisa nesta área reside no fato de que, entre 1975 e 2015, o avanço tecnológico contribuiu para o aumento de 59\% da produção do valor bruto da produção agrícola como um todo. Em termos de ciência, o Brasil no que se refere ao agronegócio, vem convergindo ações entre P\&D e consequente avanços socioeconômicos, ambientais e mercadológicos. Nesse sentido, este trabalho contempla esses pilares, dado as ações das cooperativas sendo o elo entre a produção e comercialização do agronegócio.

Conclui-se que o referido framework apresenta, além de implicações acadêmicas, implicações gerenciais. A primeira, por acompanhar por meio do estado da arte a evolução das técnicas de produção, sendo um framework estruturado a partir de 29 autores especialistas em economia, estratégia e inovação, representando um recorte teórico entre os anos de 1954 e 2018. Tal framework fora ainda desenvolvido com base nas limitações espaciais e temporais das cooperativas do norte do Rio Grande do Sul. Assim, as implicações gerenciais servirão de núcleo estratégico para a gestão das cooperativas, contribuindo com o desempenho do setor. Estudos adicionais podem ser desenvolvidos, após a verificação da efetividade gerencial do mesmo, aprimorando-o para novas realidades espaciais e temporais. Logo, as implicações acadêmicas e gerenciais apresentam bidirecionalidade contemplando com a ciência social. 


\section{REFERÊNCIAS}

AGUIAR, D. R. D.; PINHO, B. J. O agronegócio brasileiro: desafios e perspectivas. Brasília: Sociedade Brasileira de Economia e Sociologia Rural - SOBER, 1998.

ANSOF, H. I. ; DECLERCK, P. R.; HAYES, L. R. Do Planejamento Estratégico a Administração Estratégica. São Paulo: Atlas, 1990.

BALLAND, P. A. Proximity and the evolution of collaboration networks: evidence from research and development projects within the global navigation satellite system (GNSS) industry, Regional Studies, v. 46, n. 6, p. 741-756, 2012.

BATAGLIA, W; SIN OIH YU, A. A sincronização da tomada de decisão estratégica com o planejamento estratégico formal. Revista de Administração Mackenzie, v. 9, n. 5, p. 82-111, 2008.

BESANKO, D. A. Economia da estratégia. Porto Alegre: Bookman, 2006.

BIRCHALL, J.; SIMMONS, R. The role and potential of co-operatives in the poverty reduction process: A research agenda. Journal of co-operative studies, v. 40, n. 1, p. 43-51, 2007.

BOSCHMA, R. Proximity and innovation: a critical assessment, Regional studies, v. 39, n. 1, p. 61-74, 2005.

CACCIOLATTI, L. A.; FEARNE, A. Marketing intelligence in SMEs: Implications for the industry and policy makers. Marketing Intelligence \& Planning, v. 31, n. 1, p. 4-26, 2013.

COORDENAÇÃO DE APERFEIÇOAMENTO DE PESSOAL DE NÍVEL SUPERIOR. Disponível em: https://www.periodicos.capes.gov.br/. Acesso em 2019.

CARVALO, R.G; ROCHA, D.T.; CARNEIRO, A.V. Indicadores Agrícolas. Juiz de Fora: Embrapa, Gado de Leite, 2019. (Ano 10, n. 71).

CERVO, L. A.; BERVIAN, A. P.; SILVA, R. Metodologia científica. 6. ed. São Paulo: Pearson Prentice Hall, 2007.

CHADDAD, F. R.; COOK, M. L. Understanding new cooperative models: an ownershipcontrol rights typology. Applied Economic Perspectives and Policy, v. 26, n. 3, p. 348-360, 2004. 
COHEN, T. R; INSKO, C. A. War and peace: possible approaches to reducing intergroup conflict". Perspectives on Psychological Science, v. 3, n. 2, p. 87-93, 2008.

COHEN, W.; LEVINTHAL, D. A. Absorptive capacity: a new perspective on learning and innovation. Administrative Science Quarterly, v. 35, n. 1, p. 128-152, 1990.

COOK, M. L. The future of US agricultural cooperatives: A neo-institutional approach. American journal of agricultural economics, v. 77, n. 5, p. 1153-1159, 1995.

CORTE, V. F. D.; WAQUIL, P.D.; STIEGERT, K. Wheat Industry: Which Factors Influence Innovation? Journal of Technology Management \& Innovation, v. 10, p. 11-17, 2015.

COSTA, R. P.; PORTO, S. G.; SILVA, B. T. A. Evaluation of the Determinants of Technological and Managerial Results of Cooperability in Brazilian Multinationals. Journal of Technology Management \& Innovation, v. 8, n.4, p.4-23, 2013.

COSTA, R. P.; PORTO, S. G.; GALINA, R. V. S; PISCOPO, R. M.; MACCARI, A. E. Global Organization of Innovation and Cooperability in Brazilian Multinationals. Journal of Technology Management Innovation, v. 12, n.1, 2017.

DAGNINO, G. B.; LEVANTI, G.; MINA, A.; PICONE, P. M. Interorganizational network and innovation: a bibliometric study and proposed research agenda". Journal of Business \& Industrial Marketing, v. 30, n. 3/4, p. 354-377, 2015.

DAVIS, M. H. Measuring individual differences in empathy: evidence for a multidimensional approach. Journal of Personality and Social Psychology, v. 44, n. 1, p. 113-126, 1983.

DAVILA, A.; FOSTER, G. Management control Systems in Early-Stage Startup Companies. The Accounting Review, v. 82, n. 4, p. 907-937, 2007.

DENZIN, K. N.; LINCOLN, Y. Collecting and interpreting qualitative materials. 3. ed.. London: Sage Publications, 2003.

DI STEFANO, A.; GAMBARDELLA, G.; VERONA, G. Technology push and demand pull perspectives in innovation studies: Current findings and future research directions. Research Policy, v. 41, n. 8, p. 1283-1295, 2012.

DODGSON, M.; ROTHWELL, R. Technological collaboration and innovation. In DODSON M.; ROTHWELL, R. (Eds.) The Handbook of Industrial Innovation, Cheltenham: Edward Elgar Publishing, p. 285-292, 1994. 
EMPRESA BRASILEIRA DE PESQUISA AGROPECUÁRIA. Visão 2030: o futuro da agricultura brasileira. Brasília: Embrapa, 2018.

FILLION, J. L. Empreendedorismo: empreendedores e proprietários-gerentes de pequenos negócios. Revista de Administração, v.34, n.2, p. 5-28, 1999.

HEAVEY, C.; SIMSEK, Z. Top management compositional effects on corporate entrepreneurship: The moderating role of perceived technological uncertainty. Journal of Product Innovation Management, v. 30, p. 837-855, 2013.

GAZDECKI, M. Factors of business relationships change in Agribusiness inputs distribution channel. IMP Journal, v. 12, n.3, p. 567-582, 2018.

GELDES, C.; HEREDIA, J.; FELZENSZTEIN, C.; MORA, M. Proximity as determinant of business cooperation for technological and non-technological innovations: a study of an agribusiness cluster. Journal of Business \& Industrial Marketing, v. 32, n. 1, pp. 168-179, 2017.

GINSBERG, A. Connecting diversification to performance: A sociocognitive approach. Academy of Management Review, v. 15, p. 514-535, 1990.

GOODALE, J.; KURATKO, D.; HORNSBY, S. J.; COVIN, G. J. Operation management and corporate entrepreneurship: the moderating effect of operations control on the antecedents of corporate entrepreneurial activity in relation to innovation performance. Journal of Operations Management, v. 29, p.116-127, 2011.

GUIMARÃES, K. M. S; AZAMBUJA, R. L. Empreendedorismo High Tech no Brasil: condicionantes econômicos, políticos e culturais. Sociedade e Estado, v. 25, n.1, p. 93-121, 2010.

HERNANDEZ, L. K. A.; MESA, F. A.; SCHACHTER, E. M. Team collaboration capabilities as a factor in startup success. Journal of Techonology \& Innovation, v. 13, n. 4, 2018.

INTERNATIONAL CO-OPERATIVE ALLIANCE. Co-operative identity, values \& principles. Disponível em: https://www.ica.coop/en/cooperatives/cooperative-identity. Acesso em: 20 mar. 2019.

LANDER, H. G.; REINSTEIN, A. Corporate governance and economic value alignment. International Advances in Economic Research, v. 11, p. 433-447, 2005. 
LAMORE, R.; BERNSTEIN, R. R.; SCHWEITZER, H. J.; LAWTON, L. J.; RORABACK, E.; PERUSKI, A.; DYKE, M.; FERNANDEZ, L. Arts and crafts: critical to economic innovation. Economic Development Quarterly, v. 27, n. 3, 2013.

LÖBLER, M. L.; HOPPEN, N. Uso da Informação e Estratégias de Decisão na Interação com um SAD. Anais[...]. Brasília: XXIX ENANPAD, 2005.

MINTZBERG, H .; LAMPEL, J.; QUINN, J. B.; GHOSHAL, S. The process of strategy, concepts, contexts and selected cases. 4. ed. Porto Alegre: Bookman, 2006.

MISES, L. V. Ação Humana: um tratado de economia. Campinas: Vide Editorial, 2015.

MOONEY, P. H. Democratizing rural economy: Institutional friction, sustainable struggle and the cooperative movement. Rural Sociology, v. 69, n. 1, p. 76-98, 2004.

MOURA, C. C.; MADEIRA, J. M.; DUARTE, P. A. F.; CARVALHO, J.; KAHILANA, O. Absorptive capacity and cooperation evidence in innovation fron public policies for innovation. International Journal of Innovation, v.11, n.1 p. 2-19, 2019.

MORÉ, O. P. R.; GONÇALO, R. C.; FIATES, S. G. G.; ANDRADE, O. R. C. Governance structure promoting innovation: an exploratory study in Brazilian habitats. Journal of Technology Innovation, v. 13, n.4, 2018.

MUKHERJEE, K.; UPADHYAY, D. Effect of mental construals on cooperative and competitive conflict management styles. International Journal of Conflit Management. v. 30, n.2, p. 202-226, 2019.

NELSON, R.; WINTER, G. S. An evolutionary theory of economic change. Cambridge: Belknap of Harvard University, 1982.

OLIVEIRA, D. P. R. Manual de gestão de cooperativas: uma abordagem prática. 7. ed. São Paulo: Atlas, 2015.

HÅKANSSON, H. International Marketing and Purchasing of Industrial Goods: an Interaction Approach. Nova York: John Wiley \& Sons, 1982.

KAWAMOTO, T. C.; SPERS, G. R. A systematic review of the debate and the researchs of disruptive innovation. Journal of Technology Management \& Innovation. v. 14, n.1, 2019.

PENROSE, E. Strategy/Organization and the metamorphosis of the large firm. Organizational Studies, v. 29, p. 1.117-1.124, 2008. 
SALAS, E.; ROSEN, M. A.; BURKE, C. S.; GOODWIN, G. F. The wisdom of collectives in organizations: an update of the teamwork competencies. In SALAS, E.; GOODWIN, G.F; BURKE, C.S. (Eds.). Team Effectiveness in Complex Organizations: CrossDisciplinary Perspectives and Approaches, Nova York: Routledge/Taylor \& Francis Group, p. 39-79, 2009.

RIBEIRO, K. A.; NASCIMENTO, D. C; DA SILVA, J. F. B. A importância das cooperativas agropecuárias para o fortalecimento da agricultura familiar: o caso da Associação de Produtores Rurais do Núcleo VI Petrolina/PE. Teoria e Evidência Econômica, ano 19, n. 40, p. 77-101, 2013.

LAVIE, D.; ROSENKOPF, L. Balancing Exploration and Exploitation in Alliance Formation. Academy of Management Journal, v. 49, 797-818, 2006.

SCHUMPETER, J. A. History of economics, Oxford University Press, 1954.

LANGFIELD-SMITH, K. A review of quantitative research in management control systems and strategy. In C. S. CHAPMAN, A. G.; HOPWOOD; M. D. SHIELDS (Eds.). Handbook of management accounting research, Oxford: Elsevier, p. 753-783, 2007.

NOVKOVIC, S. Defining the co-operative difference. The Journal of Socio-Economics, v. 37, n. 6, p. 2168-2177, 2008.

ORTMANN, G. F.; KING, R. P. Agricultural cooperatives I: History, theory and problems. Agrekon, v. 46, n. 1, p. 18-46, 2007.

PIVOTO, D.; WAQUIL, P. D.; OLIVEIRA, C. A. O.; MORES, G. V.; CORTE, V. F. D. Gestão de organizações cooperativas: o problema do portfólio em cooperativas agropecuárias do Rio Grande do Sul. Revista de Gestão e Organizações Cooperativas, v. 1, p. 5162, 2014.

ROSA, S.; MEDEIROS, J. J. Processo decisório estratégico em cooperativas de crédito brasileiras. Anais [...]. Rio de janeiro: XXXI Encontro da ANPAD, 22 a 26 de setembro 2007.

SCIELO. Disponível em: https://search.scielo.org/ Acesso em 2019.

SIMON, A. H. As ciências do artificial. Coimbra: Almedina, 1981.

TEECE, J. D. Business Models, Business Strategy and innovation. Long Range Planning, v. 43, p.172-194, 2010. 
TIDD, J.; BESSANT, J.; PAVITT, K. Gestão da inovação. 3. ed. Porto Alegre: Bookman, 2008.

TORTORIELLO, M. The social underpinnings of absorptive capacity: the moderating effects of structural holes on innovation generation based on external knowledge. Strategic Management Journal, v. 36, n. 4, p. 586-597, 2014.

ZAHRA, S.; SAPIENZA, H.; DAVIDSSON, P. Entrepreneurship and Dynamic Capabilities: A Review, Model and Research Agenda. Journal of Management Studies, v. 43, n.4, p. 917-955, 2006.

VERGA, E.; SILVA, S. F. L. Empreendedorismo: evolução histórica, definições e abordagens. Revista de Empreendedorismo e Gestão de Pequenas Empresas, v. 3, n. 3, p. 3-30, 2014.

VERHEES, F. J. H. M.; LANS, T.; VERSTEGEN, J. A. A. M. The influence of market and entrepreneurial orientation on strategic marketing choices: the cases of Dutch farmers and horticultural growers. Journal on Chain and Network Science, v. 12, n. 2, p. 167-180, 2012.

VIETA, M. Living economics: Canadian perspectives on the social economy, cooperatives, and community economic development. Canadian journal of nonprofit and social economy research, v. 1, n. 1, 2010.

WAAL, A.; MEINGAST, A. Applying the high performance organisation framework in the horticulture and greenhouse sector. Measuring Business Excellence, v. 21, n. 2, p. 136-151, 2017.

WALTERS, D.; RAINBIRD, M. Cooperative Innovation: a value chain approach. Journal of Enterprise Information Management, v. 20. n. 5, p. 595-607, 2007.

WILSON, M.; SHAW, L.; LONERGAN, G. Our story: Rochdale pioneers museum. 2012. 
